\title{
VARIOUS DIMENSIONS OF TRUST IN THE HEALTH CARE SYSTEM
}

\author{
Maciej Bartłomiej Rogala', Ewa Donesch-Jeżo ${ }^{2}$ \\ 1 DEPARTMENT OF HEALTH POLICY AND MANAGEMENT, INSTITUTE OF PUBLIC HEALTH, FACULTY OF HEALTH SCIENCES, \\ JAGIELLONIAN UNIVERSITY, MEDICAL COLLEGE, CRACOW, POLAND \\ 2 FACULTY OF PSYCHOLOGY AND HUMANITIES "ANDRZEJ FRYCZ MODRZEWSKI" CRACOW UNIVERSITY, CRACOW, POLAND
}

\section{Abstract}

Trust is one of the most important factors in building effective and long-lasting relationships in the entire healthcare sector. Trust becomes a valuable ally in situations of high risk and uncertainty as well as the increasing complexity of inte tasks that accompany the daily work of all medical professionals, especially paramedics, due to the nature of their work in the medical rescue system. Mutual trust is the basis of social capital thanks to which it is possible to achieve mutual benefits, easier to coordinate activities, create new quality and solutions through cooperation or strong ties. It is a kind of glue that binds various organizational and system links, thanks to which it is easier to plan and introduce necessary improvements and changes within individual medical units or the entire health care system. The high degree of trust increases the quality of clinical communication with the patient and the effectiveness of medical care and strengthens the employees' motivation and willingness to cooperate. Creating social capital based on trust is in the interest of all internal and external stakeholders of the entire health care system, thanks to which joint, cross-sectoral projects for health in the public dimension are possible.

The aim of the article was, on the one hand, to systematize the theoretical and practical knowledge related to the multidimensionality of trust in the health care system, but on the other, to identify and analyse the factors building trust that should be discussed and, above all, applied in everyday professional practice and social space of all employees of the health care system. with particular emphasis on paramedics.

\section{Key words}

health care system trust, interpersonal and impersonal trust, social capital, cooperation based on trust, medical professionals

\section{INTRODUCTION}

Trust has a very complex nature and is considered in various dimensions, which results in difficulties in the elaboration of one unambiguous conceptualization. A concept of trust is usually analysed on different levels: interpersonal or impersonal/social and micro- or macro-levels [1,2].

Trust is a subject of theoretical and empirical research in many scientific disciplines and areas of social activities. Its role is widely presented in both social and health sciences and medicine. Trust has been defined by many authors and its importance has been investigated in many contexts associated with individual subjects and public health. Among terms that are attributed to trust as having similar or close meaning are hope, confidence, trustworthiness, and faith. Different scientific interpretations of the concept of trust do not allow for one universal explanation, underlying interdisciplinary approach, and wide application of trust in simple individual interactions and all kinds of complex social relationships, which is especially important in all groups of health care professionals in their relations with patients and contacts both with individual organizations and the whole health sector [2-5].

Theoretical approaches, conceptual analyses, and empirical data concerning trust emphasize a broad understanding of this concept which requires different criteria for its classification. The significance of trust in social life is manifested in various dimensions and in many scientific disciplines such as sociology, management, economics, ethics, psychology, public health, and medicine. Numerous definitions of trust in both general and medical context stress its feature of "optimistic acceptance of the vulnerable situation in which the truster believes that the trustee will care the truster's interests," and in a broader sense, trust is described as "the lubricant of the social system". Moreover, studies confirm the importance of mutual trust as a factor in improving cooperation and reducing the need for monitoring [6-8].

Research also suggests a significant contribution of trust to the development of cooperative or collaborative relationships in many aspects of social interactions [9]. These features seem to play a significant role in an everyday work of paramedics where relations 
based on interpersonal and systemic trust build positive relations and social networks, shape collaboration, which usually results in higher motivation. Conversely, the lack of trust or its low level may hinder or prevent effectiveness, collaboration, and even not lead to planned organizational or systemic change.

For many years practical implications of trust in the health care system have been widely studied and discussed. Results of research and clinical practice revealed a positive role and influence of trust in all health care relationships and settings and a strong link between patient's trust in health care professionals and health outcomes [10-12]. What is more, trust in health care is regarded as one of the key factors which may support effective treatment and create a foundation for patient-centred care [13, 14]. Research findings require not only their implementation but also the dissemination of the knowledge and benchmarking of good practices on how to build trust-based relationships among all health care professionals including paramedics working in the emergency system.

\section{REVIEW AND DISCUSSION}

\section{DIFFICULTIES WITH UNAMBIGUOUS TRUST EXPLANATION}

Among known and common views on trust cited in literature which emerged from theoretical analyses are definitions proposed by modern and influential representatives of social sciences such as Erik H. Erikson, Anthony Giddens, Michel Foucault, Francis Fukuyama, Niklas Luhmann, James Coleman, Martin Hartmann, Russell Hardin, Diego Gambetta, and Piotr Sztompka. All of them have made a significant contribution to the knowledge about trust and understanding of this concept, which in consequence created an area for studies for many contemporary researchers analysing the influence of trust on different levels of relationships in the whole health care system. The conceptualizations of trust offered by the above-mentioned authors often contained terms related to it such as "leap of faith", "willingness to be vulnerable or to take a risk", "a key component of social capital", "a component of power-knowledge and true-telling" or "belief in the responsibility for others". The results of many studies suggest that it is difficult to elaborate one general definition of this concept because trust has been investigated from many perspectives and in many disciplines. This resulted in many theoretical approaches derived from different backgrounds. Among these approaches, an especially interesting and important for the goals of this paper seems to be the one treating trust as a ba- sic component of social capital as a kind of glue that bonds and bridges distinctions, strengths weak ties, creates a sense of community between disconnected networks and forms a kind of social fabric of connection and collaboration [15].

Although there are many differences in conceptualisations and theories dealing with trust, they all have one common element - all of them emphasize the distinct role of trust in the functioning of societies including health care systems. Looking for similarities in the definitions, it needs to be pointed that trust can develop from three main factors: experience, information/knowledge, or an expected outcome, all of which are based on the trusting relationship. Trust is usually built on some uncertainty and is associated with risk because the outcome of a trusting relationship cannot is unpredictable $[2,16]$.

In this place, it is worthy to present the approach to this concept proposed by researchers engaged in studies on trust in medical institutions and health care systems. On the one hand, this approach emphasizes the general and multidimensional nature of trust and on the other hand, the important role of trust in health care. The approach has been named the "optimistic acceptance of a vulnerable situation" which indicates that the trustee believes that the trustor will take care of the trustee's interests or will act in his/her best interests at heart $[1,7,8]$.

Other known classical trust conceptualization depicts "the willingness of a party to be vulnerable to the actions of another party based on the expectation that the other will perform a particular action important to the trustor, irrespective of the ability to monitor or control that other party" [17] and "a state involving confident positive expectations about another's motives with respect to oneself in situations entailing risk" $[18,19]$.

A common situation associated with trust is a kind of relationship between minimum two individuals which is described as "A trusts B to do - or not to do $-\mathrm{X}$ ". A trusting relationship is also described between groups as "A - group, B - group" of people, organisations, institutions, companies. Trust is based on relations and is developed by the exchange of "truthful" information without which trust cannot be built. It is emphasized that truthful information is especially important in health care, as it allows, for example, to make an informed decision in the patient consent process [16]. The studies on trust show that it applies to people, social or organizational systems, that is, to individual persons, groups, social entities, relations between people and groups [20]. 
Assuming that trust is regarded as a kind of optimistic acceptance of a vulnerable situation that focuses on the positive intentions of the trusted individual or organisation, it should be taken into attention that this concept is always associated with some uncertainty regarding activities, behaviours, and attitudes that may or may not occur. Trust allows people to undertake present or future decisions based on previous experience and the knowledge of the past, which minimizes the risk associated with undertaking actions. A part of researchers suggest that trust occurs only in risky situations. In situations without risk, we can speak about confidence or expectation rather than trust $[1,21,22]$. The outcomes of studies indicate that:

1. trust is associated with uncertainty;

2. trust is accompanied by fear of losing something valuable;

3 . trust concerns a partner whose attitudes and actions cannot be fully controlled [23].

Some researchers distinguish 5 categories of trust which make a continuum - from distrust (deterrencetrust), low trust (calculus-based trust), confident-trust (knowledge-based trust), high/strong trust (relational-based trust) to complete trust (identification-based trust). This classification includes five fundamental types of experiencing trust based on its intensity [19].

The very act of trusting someone elicits a reciprocal reaction, which favours building relationships on both sides - that of a truster and a trustee. Although trust is a consequence of the assessment of the reliability of the relationship between partners, it is not an objective phenomenon, as it is established on individual perception. As a rule, trust may result from the evaluation, direct or indirect, of the data reflecting the trusted person's attitudes, behaviour, characteristics, and qualities. Trust is dynamic and is conditioned by the dynamics of relations between the two parties participating in interactions. The tendency to trust others is individual. In interpersonal trust, the credibility of the other person is the basis of trust, and because it is connected with the perception of another person, it can be subjected to evaluation. Establishing credibility occurs through certain determinants which depict someone credible and someone unreliable based on specific features, characteristics, or behaviours. Researchers of the problem of trust have put forward many determinants of credibility, and it is impossible to create a closed list of them, since they include a variety of concepts such as motivation, personality traits, competencies, honesty, predictability, intentions, abilities, consistency, openness, keeping promises and reliability $[19,24,25]$.
In the debates about trust conceptualizations, the distinction has been made between two opposites on one continuum - trust and distrust (a lack of trust). Later this distinction between trust and distrust has been changed and currently, trust and distrust are regarded as two separate constructs that differ from each other by contrasting characteristics and determinants. One of the approaches describes trust and distrust in terms of disposition. Distrust is not only the absence of trust but is also seen as "an actor's assured expectation of intended harm from the other" while "disposition to trust means the extent to which one displays a consistent tendency to be willing to depend in general on others across a broad spectrum of situations and persons" [26, 27]. Trust is based on "confident positive expectations regarding another's conduct" whereas distrust is based on "confident negative expectations regarding another's conduct" [28]. Researchers of this problem also distinguish a culture of trust and culture of distrust. In this perspective culture of distrust is characterised by "a pervasive, generalized climate of suspicion" that in consequence can lead to alienation and passivism [29].

Empirical studies on trust indicate a relationship between trust, and monitoring, and controlling. Investigations have provided evidence that there is an inverse association between trust and monitoring and controlling. On the one hand, the primary value of trust is linked to the reduction in monitoring and controlling, which means that there is no need to monitor and control the activities and behaviour of a fully trusted partner. On the second hand, reduction in monitoring and control is associated with the increase of trust and may positively influence joint working, open communication, problem solving, and gradual improvement that can be used in project management. The pointed out role and effects of trust will be presented in the next parts of this paper [9].

\section{MULTIDIMENSIONALITY OF TRUST IN ORGANISATIONS}

The multidimensional theory of trust does not facilitate the resolution of problems on how to explain its wide application and importance. Trust is described as a complex phenomenon associated with many theories and approaches. As a construct, trust has a strong background in sociology but its role is also deeply discussed in psychology, economics, management, and philosophy. Its role in individuals, organisations and other social processes is widely discussed. Researchers from many disciplines treat trust as a factor contributing to many benefits in interpersonal and social interactions and emphasize its role in health care organizations [21]. These findings 
are a subject of many analyses and scientific studies on how to use the knowledge and the gained interdisciplinary experience in organizational processes in the everyday work of professionals in health care systems [7].

Described in this paper difficulties in generalising trust as a concept are strongly associated with its function in societies. Human future-oriented actions are accompanied by uncertainty or risk. Uncertainty is connected with contingent actions of others - we never know how the partners in our interactions and social relationship will react. Based on their rational calculations, people estimate the amount of risk they can take or, in other words, to what extent they can trust others $[21,30]$.

Regardless of the differences in approaches to trust, problems in agreeing on an unambiguous definition of this concept or the scientific discipline which should examine the effects of trust, it is assumed, and seems to be a common point of view, that trust plays an important role in every organization, including health care institutions in which health professionals work, including rescuers performing their professional functions across the entire medical rescue system. Trust is an indispensable condition for building and continuity of various relationships and is particularly important in the professional sphere of man, as it effectively determines the activity and cooperation between people, institutions, or entire systems, which is particularly important in the sphere of health protection $[3,4,6,16,24]$.

Three levels of trust emerge: interpersonal trust in a given organization, a person's trust in the organization, and trust between organizations. Each of these levels plays a significant role in the health care system $[7,24]$.

Many studies show an important role of trust in organizations in the context of interpersonal behaviour and management. As a rule, trust arises in risky situations and is therefore assumed to be related to the management of uncertainty or risk. It has been proven that a high degree of trust is necessary to engage employees effectively in co-operation and enhance the effectiveness of the organization. Besides, trust at the interpersonal level allows us to increase trust at the organizational level [23].

As it seems, the results of studies on trust should be taken into account by all health care organizations, especially those that operate in an acute mode and admit patients in emergences performing their tasks in the face of high risk and crises, where trust is or should be one of the cornerstones of their professional business.
Studies indicate the importance of trust as an integral factor of creating a business or professional relationship and affecting the effectiveness of the strategic management process in the context of communication with external partners, with employees and as a factor that can decrease the costs of transactions. Moreover, trust may turn valuable in exchange relationships. [4, 23].

The research results indicate the crucial role of leaders and managers as people who should build a long-term partnership with external and internal stakeholders and systematically participate in building a dialogue with them. Trust strengthens cooperation and is one of the key elements of the assessment of internal relations between managers and employees. A high level of trust in an organization translates into better information exchange between people, better engagement of co-workers, greater loyalty and innovation as well as willingness to cooperate $[9,23,31]$.

It is also assumed that trust is a critical factor influencing three important areas in organizations such as coordination of activities, risk-taking, and facilitates mobilization of resources for productive activities. Trust is important in all institutions and essential in the context of organizational change because it is usually connected with increased complexity, uncertainty, and risk. In situations of change, people always need at least a minimum of trust in their leaders who are responsible for planning, implementation, and evaluation of change in the organization. Additionally, trust strongly influences employees' compliance with managers' decisions, which is important not only in everyday activities but also at the stage of formulation and development of strategies and change initiatives. In this meaning, trust between managers and employees may create a kind of interface between the development and realization of strategic initiatives. Empirical findings also suggest that some highly successful organizations acting in high turbulence environment were able to make better and faster decisions because of effective and multimodal communication channels based on trust $[9,17,20$, 23, 24, 31].

While trust shapes the initiation and development of organizational relationship in a workplace, distrust works in the opposite direction having an impact on the dissolution of these relations. Structures in organizations based on personal networks and friendships are more vulnerable to the detection of relevant environmental stimuli and the mobilization of resources that can be used during change strategies. Conversely, weak ties in organizations may result in the 
inability to identify serious signals from the environment and obstruction of the mobilization of resources necessary to plan, organize, and implement some organizational change $[32,33]$.

Theoreticians and researchers of this problem alike indicate that among many benefits to organizations, trust supports the development of important initiatives and helps solve problems connected with an open exchange of information and knowledge, process of inter-organizational learning, conflicts between organizations, coordinating economic activities, organizational changes, and adaptation. Moreover, trust is regarded as a factor positively influencing a significant reduction of transaction costs, creation of joint strategies, facilitation of investments in a long-term perspective, and limitation of opportunism $[23,24,32,33]$. The dependencies described in this part of the article apply to organizations both in a private and public sector, they are cross-sectoral and can be successfully used in all health care institutions which employ paramedics.

\section{ROLE OF TRUST IN THE HEALTH CARE SYSTEM}

Trust in healthcare is generally defined as a set of patient's expectations from the healthcare system to help them cure their medical problem. These expectations include appropriate diagnosis, correct treatment, non-exploitation, interest in the patient's welfare and transparent disclosure of information. Collected data about trust indicate that it plays a substantial role in improving the performance of the healthcare system which is defined by the WHO "as comprising all the organizations, institutions and resources that are devoted to producing health actions. A health action is defined as any effort, whether in personal health care, public health services or through intersectoral initiatives, whose primary purpose is to improve health" [34-37].

The fundamental function of trust in health care system derives from the fact that the health care provision is based on uncertainty and unpredictability and its erosion may lead to the breakdown of cooperation in the area of health $[3,35]$.

Practical application of trust is widely analysed in health care sectors which results in numerous scientific research in the field of medicine and public health examining the importance of this concept in various areas and explaining its significant role in all health care relationships. Importance of trust in health sector refers to two basic aspects of trust: interpersonal trust between individuals and social trust between institutions which are the subject of many analyses and empirical studies. Results of theoretical and practical research indicate that trust can be treated as one of the important factors in the improvement of performance in the health care system. Moreover, trust is regarded as one of the main ingredients of effective, high-quality health care. The findings also recognize the influence of trust on health outcomes [7, 8, 38, 39].

Trust between different actors in the healthcare triangle (between insured and insurers, healthcare insurers and providers, healthcare providers and patients) in the healthcare system facilitates the cooperation that is necessary to reach the goals of healthcare systems. Although a majority of research on trust is focused on interpersonal trust between patients and healthcare providers, it should be mentioned that interpersonal trust may also affect the functioning of healthcare systems and their components. The institutional trust may be observed in health care providers in general, the medical professionals, hospitals and other medical institutions. Trust between the actors in the healthcare triangle may affect the effectiveness, efficiency and the longevity of these relationships, quality of care, and overall success of policies [1, 40, 41].

As has been mentioned earlier, trust is distinguished into interpersonal trust including the day-today interactions between individuals, and social trust which may build through the collective actions of social institutions. In this meaning, trust is categorised according to the object of trust and may be analysed as trust in individual healthcare professionals and trust in healthcare institutions [7, 8, 34].

Trust in the healthcare system is understood as a combination of trust in the healthcare providers (called as an individual trust) and trust in the healthcare institutions (called as institution trust) which create a conceptual model named as health care system trust. The main elements of this system which influence trust include sociodemographic factors, previous experience with providers and institutions, cultural factors and media influence. Among the benefits of health care system trust are: better adherence, improved health-seeking pattern, increased health care utilisation and improved communication. Researchers analysing the influence of trust in health care emphasize that the health care system trust should be characterized by transparency in the transactions between healthcare professionals and individuals, effective communication between them necessary to build trust, delivery of quality services by public healthcare institutions. Health care providers should be competent to correctly prevent, diagnose and treat diseases. Collected data suggest that trust in the healthcare provider (individual trust) and trust 
in the healthcare institution (institutional trust) may be regarded as one of the key factors contributing to trust in the public healthcare system [8, 16, 34, 42].

Researchers indicate that personal experience is substantial in the process of trust-building and point out the following correlation: if people have personal experience of medical staff, they trust more. Moreover, personal experience with health care system representatives encourages the transfer of trust in these representatives to trust in the wider health care system [16].

Trust in health care can be measured and is an area of many studies and analyses. Among common used scales are Public Healthcare System Trust, Multidimensional Trust in Health Care System Scale, Trust in Primary Care Physician Scale, Primary Care Assessment Survey, Trust in Physician Scale, Physician Trust Scale, Health Care Relationship Trust Scale and developed Health Care Relationship Trust Scale Revised, Health Insurance Organization Trust Scale, Whole Health System Trust Scale and the Medical Professions Trust Scale. They generally refer to individual and institutional trust and include indicators such as quality, communication, transparency, reliability, fidelity, honesty, openness, advocacy and administrative and clinical competence $[2,8,16,43]$

Trust as a multidimensional construct cannot be measured directly and it is difficult to measure it due to its complexity. The majority of used scales measure trust in different components of the healthcare system and only a few asses this system as a whole. According to recommendations, measuring trust in the healthcare system facilitates building a system which may be more trustworthy and based on better health outcomes [8].

Studies show an influence of trust on health outcomes is connected with patient's satisfaction, adherence to treatment and continuity of care with a health care provider. Moreover, trust encourages patients to access health care as well as results in a higher quality of interaction, and lower transaction costs in the health system. Additionally, trust enables health care providers to encourage necessary behavioural changes and may grant patients more autonomy in decision-making about treatment. It is worthy to emphasize that patients having trust in health care professionals reported more beneficial health behaviours, fewer symptoms and higher quality of life and were more satisfied with treatment. Collected data suggest that relationships based on trust are especially important in chronic disease management as a factor contributing to adherence with medical advice and is perceived as a crucial component of effective therapeutic procedures [1, 2, 37, 44-47].
Research data show that there is a kind of connection between trust and satisfaction. On the one hand, trust has the same functional attributes as satisfaction and on the other hand, satisfaction is based on an assessment of past events, while trust is based on a forward-looking evaluation of an on-going relationship $[1,39,48]$. Some empirical research also present a specific problem of the relation between trust and dependence especially in emergencies when patients have no choice (dependence) and "have to trust" in the medical system and their medical members. Responses of participants taking part in the research suggest the dependence of these patients on the medical emergency system rather than a trust which is based on choice. The problem of a clear distinction between trust and dependence remains unresolved because any emergency circumstances are characterized by immediate risk and an urgent need for medical attention which can be provided only by medical professionals. In these situations, patients who were participants of the research despite earlier negative experiences with the medical system and health professionals, indicated that they had no other choice but to trust in medical professionals in emergency situations because they were dependent on them. They passively accepted performed actions in situations of health or life risk and patients' compliance or submission resulted not from the trust but rather dependence [21].

Degree of competence presented by medical professionals is regarded as a key factor affecting trust. Studies indicate that patient's experience can be associated with the expectation of care and appropriate cure and with the nature of the relationship with a particular health care provider including concern and empathy. On the other hand, some findings suggest that trust may arise when patients are convinced about medical professionals' competencies which include $[3,49]$ :

- carrying out appropriate tests to confirm the diagnosis before treatment;

- consistent diagnoses between colleagues;

- calling a senior colleague promptly when the case requires it;

- ability to identify a serious case through triage and prioritise treatment accordingly;

- consistent follow-up of patients to check progress.

The importance of trustworthiness of individual health professionals and healthcare institutions (hospitals, health centres, emergency system) and forms of professionalised knowledge which are crucial for health systems to function in the interest of society have been emphasized [47]. 
Continuing the theme of professional competences, it is worthy to note an example of research measuring trust among health care professionals in Poland - a study comparing the level of trust in physicians, nurses and paramedics which indicated in patients' opinion important advantages and disadvantages of each of these occupational groups. The questionnaires included four areas of questions such as contact with the patient (good/wrong), manual skills (possessing/ lack), diagnosis/therapy (accurate/wrong) and level of knowledge (high/low). In respondent's assessment, the best advantages of physicians were the accuracy of the diagnosis and therapy and high level of knowledge and the best advantages of nurses were good contact with a patient and manual skills and in case of paramedics - manual skills and high level of knowledge. The respondents pointed that among the biggest disadvantages of physicians were wrong diagnosis/therapy and bad contact with patients, the biggest disadvantages of nurses were bad contact with a patient and low level of knowledge and of paramedics low level of knowledge and bad contact with a patient [50].

Although some researchers analysing the importance of trust in health care system have primarily focused on the relationship between physicians and patients, over time, the issue of trust also began to be raised in relationships between patients and health care teams, particular health care providers and organizations as whole systems and also managers supervising health care units $[3,46]$. In this meaning, research indicate also benefits of trust between managers and medical professionals which reduces the need for monitoring and may result in bigger job satisfaction and staff retention as well as higher efficiency of organisational performance.

In the face of uncertainty and risk at various levels (low, medium or high), there is usually uncertainty related to the motives, intentions or future actions of other people. These circumstances strengthen the need for trust, and in health care - as suggested by the results of various studies - it manifests itself through embracing confidence in competence understood as skills and knowledge and whether the trustee will work in the best interests of the trustor [17, 46, 51].

Collected and interpreted data about trust emphasize the fact that successful and sustainable cooperation results in a foundation of trust and reciprocity as well as the reduction of complexity and risk [52]. These values seem to be invaluable and it is worth considering how to put them into practice or improve their functioning in the face of the challenges encountered by modern medicine, health care systems in various countries, as well as people who shape it daily, being its integral part as medical professionals.

\section{CONCLUSIONS}

The article shows the multidimensionality of trust and the role it plays in the entire health care system. The collected cross-sectional data on the importance of the concept research proves that mutual trust improves long-term and effective cooperation, reduces the need for monitoring, and triggers reciprocity. Moreover, it is the basic factor that builds social capital and a kind of glue that binds various organizational and system links, thanks to which they can efficiently implement their goals and tasks.

Trust is especially important in situations where the degree of risk, uncertainty, and complexity increases, as it allows us to reduce them and deal with them better. The fact that people can trust each other in situations requiring effort, cooperation, stress, and challenges has a positive effect on their relationships, which in such situations can strengthen and further develop positively. Research shows that a high degree of interpersonal trust translates into increased trust in the activities of health care units and the entire health care system, which seems to be a positive effect of shaping a culture based on trust ties, not only in the interpersonal but also in the wider context - general society.

It should be noted here that the professional duties of paramedics working in the medical rescue system are particularly burdened with risk and the occurrence of uncertainty as well as complexity. That is why building a climate of trust is so important both concerning patients who need emergency medical care, as well as members of the medical rescue team with whom they work daily, as well as other employees and superiors in the organization and the entire medical rescue system.

Trust in health care can be assessed at the three mentioned levels: interpersonal, organizational, and systemic. Thanks to trust at the interpersonal level, especially concerning the relationship between patients and medical professionals, a better level of adherence and compliance to treatment is observed, as well as a higher level of satisfaction with medical care, greater willingness to use health care services, including the continuation of care with a specific health care provider, as well as the perceived higher health-related quality of life.

Thanks to trust at the organizational level, it is possible to create joint plans aimed, on the one hand, at the improvement of a medical institution or effective change management, and on the other hand, at 
its efficient functioning. A high degree of trust in the organization is reflected in the willingness to cooperate and exchange information, greater commitment to work, loyalty of employees and innovation, and allows the mobilization of human resources in the situation of organizational change.

Thanks to trust at the system level, it is possible to establish relationships with various stakeholders and joint implementation of projects, including cross-sectoral projects, and to implement innovative strategic solutions for development in both the public and private spheres in the healthcare sector.

Trust is born out of previous experiences, information, or expected results, where there is always some element of risk and uncertainty as to whether the person, organization, or system will behave and perform as expected. This unpredictability is inherent in social interactions and is unlikely to ever go away.
Trust in the area of health is something special since it is one of the most important life values for people, and a successful fight for health protection can undoubtedly build a huge credit of trust.

However, it is worth remembering that in addition to the so-called hard competencies, which constitute the core of trust in health care, interpersonal skills also seem to be important. Establishing and maintaining relationships through open communication based on facts and reliable analysis of the situation may provide a chance to create a platform which, if it results in mutual trust - and taking into account the results of previous research on this subject - may be beneficial for all sides of this relation. Trust breeds trust, which, especially in the times of the coronavirus pandemic and many other threats to health in the individual and public dimension, seems to be an invaluable value.

\section{REFERENCES}

1. Gilson L. Trust and the development of health care as a social institution. Soc Sci Med. 2003;56(7):14531468.

2. Pearson SD, Raeke LH. Patients' trust in physicians: many theories, few measures, and little data. J Gen Intern Med. 2000;15(7):509-13.

3. Calnan M, Rowe RJ. Researching trust relations in health care: conceptual and methodological challenges-introduction. Health Organ Manag. 2006;20(5):349-5.8

4. Tschannen-Moran M, Hoy WK. A Multidisciplinary Analysis of the Nature, Meaning, and Measurement of Trust. Rev Edu Res. 2000;70(4):547-93.

5. Croker JE, Swancutt DR, Roberts MJ, Abel GA, Roland M, Campbell JL. Factors affecting patients' trust and confidence in GPs: evidence from the English national GP patient survey. BMJ open. 2013:3(5).

6. Hall M, Camacho F, Dugan E, Balkrishnan R. Trust in the Medical Profession: Conceptual and Measurement Issues. Health Serv Res. 2002;37(5):1419-439.

7. Hall MA, Dugan E, Zheng B, Mishra AK. Trust in physicians and medical institutions: what is it, can it be measured, and does it matter? Milbank Q. 2002;79(4):613-39.

8. Egede LE, Ellis C. Development and testing of the Multidimensional Trust in Health Care Systems Scale. J Gen Intern Med. 2008;23(6):808-15.

9. Meng X. The role of trust in relationship development and performance improvement. J Civ Eng Manag. 2015;21(7):845-853.

10. Lee YY, Lin JL. Linking patients' trust in physicians to health outcomes. Br J Hosp Med. 2008;69(1):42-6.

11. Safran DG, Taira DA, Rogers WH, Kosinski M, Ware JE, Tarlov AR. Linking primary care performance to outcomes of care. J Fam Pract. 1998;47(3):213-20.

12. David AS. Communicating in times of uncertainty: the need for trust. J Health Commun 2003;8(S1):1314.

13. Barry MJ, Edgman-Levitan S. Shared decision making - pinnacle of patient-centered care. New Engl J Med. 2012; 366(9):780-1.

14. Hughes JC, Bamford C, May C. Types of centeredness in health care: themes and concepts. Med Health Care Philos. 2008;11(4):455-63.

15. Leith D. Representations of the concept of trust in the literature of Library and Information Studies. Cosmopolitan Civil Societies: Interdiscipl J. 2013;5:3.

16. Gille F. Theory and conceptualisation of public trust in the health care system: Three English case studies: care.data, biobanks and 100,000 Genomes Project. PhD thesis, London School of Hygiene \& Tropical Medicine; 2017.

17. Mayer RC, Davis JH, Schoorman FD. An integrative model of organizational trust. The Academy of Management Review. 1995;20(3):709-34. 
18. Boon SD, Holmes JG. The dynamics of interpersonal trust: resolving uncertainty in the face of risk, In: Hinde RA, Groebel J (eds). Cooperation and prosocial behaviour. Cambridge: Cambridge University Press.1991, pp. 190-211

19. Dietz G, Hartog D. Measuring trust inside organisations. Person Rev. 2006;35(5):557-588.

20. Wekselberg V. Reduced "Social" in a New Model of Organizational Trust. Acad Manag Rev. 1996;21(2):333-35.

21. Meyer S, Ward PR. Reworking the sociology of trust: making a semantic distinction between trust and dependence. The Future of Sociology. Proceedings of the Australian Sociological Association conference. 2009, pp. 1-16. [https://dspace2.flinders.edu.au/xmlui/bitstream/handle/2328/26057/Meyer\%20Reworking.pdf? sequence=1. Accessed: 20.08.2020]

22. Dugan E, Trachtenberg F, Hall MA. Development of abbreviated measures to assess patient trust in a physician, a health insurer, and the medical profession. BMC Health Serv Res. 2005;5:64.

23. Sołoducho-Pelc L. The Importance of Trust in the Implementation of the Strategic Management Process. Inter J of Contemp Manag Vol. 2017;16(4):237-261.

24. Jaklik A, Łaguna M. Zaufanie w organizacji. Analiza sposobów ujęcia i modeli teoretycznych. Psychol Spol. 2015;10/4(35):369-382.

25. Sztompka P. Zaufanie. Fundament społeczeństwa. Kraków: Znak, 2007.

26. van de Walle S, Six F. Trust and distrust as distinct concepts: Why studying distrust in institutions is important. J Comparat Pol Anal Res Pract. 2013:158-174.

27. McKnight HD, Chervany NL. Trust and distrust definitions: One bite at a time. Trust Cyber Soc. 2001:27-54.

28. Lewicki RJ, McAllister DJ, Bies, RJ. Trust and distrust: New relationships and realities. Acad Manag Rev. 1998;23(3):438-458.

29. Sztompka P. Trust, Distrust and Two Paradoxes of Democracy. Eur J Soc Theory. 1998:1(1):19-32.

30. Sztompka P. Trust and emerging democracy: lessons from Poland. Inter Soc. 1996:11(1):37-62.

31. Holland P, Cooper BK, Pyman A. Trust in Management: The Role of Employee Voice Arrangements and Perceived Managerial Opposition to Unions. Hum Res Manag J. 2012;22(4):377-391.

32. Johansen ST, Selart M. Expanding the role of trust in the management of organizational change. Expanding the Role of Trust in the Management of Organizational Change. In: Lines R, Stensaker I, Langley A (eds). New Perspectives on Organizational Change and Learning. Bergen: Fagbokforlaget. 2014:259-280.

33. Dirks KT, Ferrin DL. The Role of Trust in Organizational Settings. Organ Sci. 2001;12(4):450-467.

34. Anand TN, Kutty VR. Development and testing of a scale to measure trust in the public healthcare system. Indian J Med Ethics. 2015;12(3):149-57.

35. Maynard A, Bloor K. Trust and performance management in the medical marketplace. J R Soc Med. 2003;96(11):532-539.

36. WHO. The world health report 2000 - Health systems: improving performance. Geneva: World Health Organization; 2000.[https://www.who.int/whr/2000/en/. Accessed: 08.09.2020]

37. Attar GE. Trust in the Health System: The Case of Cardiology Patients in Turkey. Atatürk Üniversitesi Sosyal Bilimler Enstitüsü Dergisi Haziran 2018;22(2):961-976.

38. Campos-Castillo C, Woodson BW, Theiss-Morse E et al. Examining the Relationship Between Interpersonal and Institutional Trust in Political and Health Care Contexts. In: Shockley E, Neal TMS, PytlikZillig LM et al (eds.) Interdisciplinary Perspectives on Trust. Towards Theoretical and Methodological Integration. 2016, pp. 99-115.

39. Robinson CA. Trust, Health Care Relationships, and Chronic Illness: A Theoretical Coalescence. Glob Qual Nurs Res. 2016;3:1-11.

40. Groenewegen P, Hansen J, de Jong JD. Trust in times of health reform. Health Policy. 2019;123(3):281-287.

41. Wilk AS, Platt JE. Measuring physicians' trust: a scoping review with implications for public policy. Soc Scien Med. 2016;165:75-81.

42. Van der Schee E, Braun B, Calnan M et al. Public Trust in Health Care: A Comparison of Germany, the Netherlands, and England and Wales. Health Policy. 2007;81(1):56-67.

43. Gopichandran V, Wouters E, Chetlapalli SK. Development and validation of a socioculturally competent trust in physician scale for a developing country setting. BMJ open. 2015;5(4):e007305. 
44. Birkhäuer J, Gaab J, Kossowsky J. Trust in the health care professional and health outcome: A metaanalysis. PLoS One 2017;7/12(2):e0170988.

45. Jin J, Sklar G, Sen Oh VM. Factors affecting therapeutic compliance: A review from the patient's perspective. Ther Clin Risk Manag. 2008;4(1):269-286.

46. Rowe R, Calnan M. Trust relations in health care: Developing a theoretical framework for the "new" NHS. J Health Organisat Manag.2006;20(5):376-96.

47. Meyer S, Ward PR, Coveney J. Trust in the health system: An analysis and extension of the social theories of Giddens and Luhmann. Health Soc Rev. 2014;17(2):177-186.

48. Hall MA, Zheng B, Dugan E et al. Measuring patients' trust in their primary care providers. Med Care Res Rev.2002;59:293-318.

49. Rachel A. What causes patients to trust medical professionals? Insights from mothers in Juba. South Sudan Med J. 2017;10(2):33-36.

50. Klepacka M, Bakalarski P. Trust of society towards selected medical professions - doctors, nurses, paramedics. Critic Care Innov. 2018;1(2):1-10.

51. Borum R. The Science of Interpersonal Trust. Ment Health Law Policy Fac Public. 2010;1.

52. Calnan M, Rowe R, Entwistle VA. Trust relations in health care: An agenda for future research. J Health Organ Manag. 2006;20(5):477-84.

ORCID AND CONTRIBUTIONSHIP *

Maciej Bartłomiej Rogala-0000-0001-8104-7617 A,B,D,E,

Ewa Donesch-Jeżo - 0000-0002-4655-8822 D,F

CONFLICT OF INTEREST

Authors declare no conflict of interest.

\section{ADDRESS FOR CORRESPONDENCE}

Maciej Rogala

Zakład Polityki Zdrowotnej i Zarządzania, Instytut Zdrowia Publicznego,

Wydział Nauk o Zdrowiu, Uniwersytet Jagielloński Collegium Medicum

ul. Grzegórzecka 20, Kraków

e-mail:maciej.rogala@uj.edu.pl

RECEIVED

ACCEPTED

19.09.2020

* Contribution: A - Work concept and design, B - Data collection and analysis, C - Responsibility for statistical analysis, D-Writing the article, E-Critical review, F-Final approval. 